\title{
Support vector machine based on power of signal for solving high-energy physics problem
}

\author{
Hind Khudheyer ${ }^{1}$, Hisham Jashami ${ }^{2}$ \\ ${ }^{1}$ University of Karbala, Department of physics, Karbala, Iraq \\ ${ }^{2}$ University of Karbala, Department of Civil Engineering, Karbala, Iraq
}

\begin{abstract}
In this paper, a new method proposed combining support vector machine (SVM) based on power of signal for high-energy physics problem. In the first stage, the high-energy physics are analyzed by using Power of signal, which produce number of sensitive features. The aim of this stage reduce the number of features and improve the quality of them. In the second stage, SVM applied to classify the new features into two categories and presented remarkable results.

(C) 2019 ijrei.com. All rights reserved
\end{abstract}

Keywords: SVM, High-Energy Physics, Power of Signal

\section{Introduction}

SVM has develop an influential technique for investigating the famous classification problem in the area of machine learning. The SVM has its basis in Statistical Learning Theory and its design is depending on the Organizational Risk Minimization standard [1]. The optimization job in SVM includes the explanation of a convex optimization problem for which an actual enjoyable theory and effectual techniques are obtainable [2]. The SVM practice has been effectively used to a huge diversity of pattern recognition problems such as., face recognition [3], confirmation and classification [4], object recognition and classification [5], handwritten character and digit classification [6], text classification [7], speech identification, etc. Machine learning (ML) approaches have been used in a number of problems in materials physics, lately [8]. These have comprised the hunt for mixes expected by theory, broadcast for new materials, classifying stable mixtures, faster forecast of material possessions, the mixture of data mining and important procedure to forecast crystal organizations, and many others. Researches in this set have applied (ML) for grouping by identifying objects and creation forecasts in the face of difficulty. Several of new researches has used Deep Neural Networks and Convolutional Neural Networks to diagnose stage changes as designs in two dimensional Ising models. Regression-based researches contain the work of Kalidindi and co-workers, who have used (ML) approaches to mine belongings depend on materials microstructure. There also has been effort on evolving the malleable harvest superficial for a material using functional regression approaches. Here, we have planned whether ML, exactly Deep Neural Networks, in mixture with extra datadriven methods, like replacement optimization, sensitivity investigation and multi fidelity modeling, can be applied to leader the forecast of hurried shapes in blends. Furthermore, power of signal is a famous signal processing function which is used to measure the power of any signal. In this paper, power of signal calculated for each period for the input data that obtained from UCI website. Moreover, the aim of power of signal is to reduce the size of dataset and extracted important features from the input data, then reduce the computational time and increase the classification rate of the SVM.

\section{Material \& Methods}

\section{$2.1 S V M$}

SVM contingent on the impression of choice airplanes that label choice bounds. A choice smooth is one that ruptures amongst a collection of belongings having numerous lessons 
associations. A figure example is offered in the Figure 1. In this example, the substances fit moreover to lessons GREEN or RED. The excruciating line labels a boundary on the correct irritated of which all substances are GREEN and to the left of which all items are RED. Any dissimilar article (white circle) plummeting to the right is proprietary, i.e., classified, as GREEN (or categorized as RED should it droplet to the left of the excruciating streak) [9].

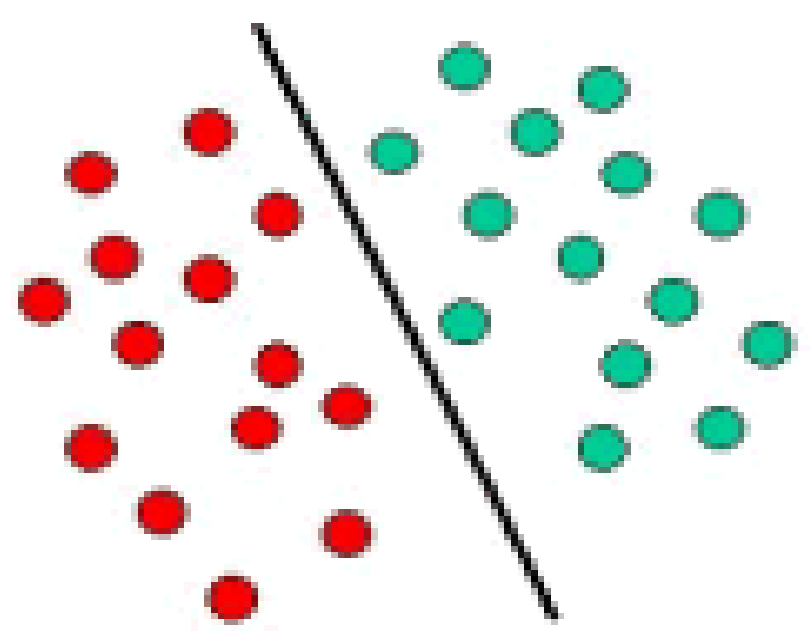

Figure 1: Classification as green and red items

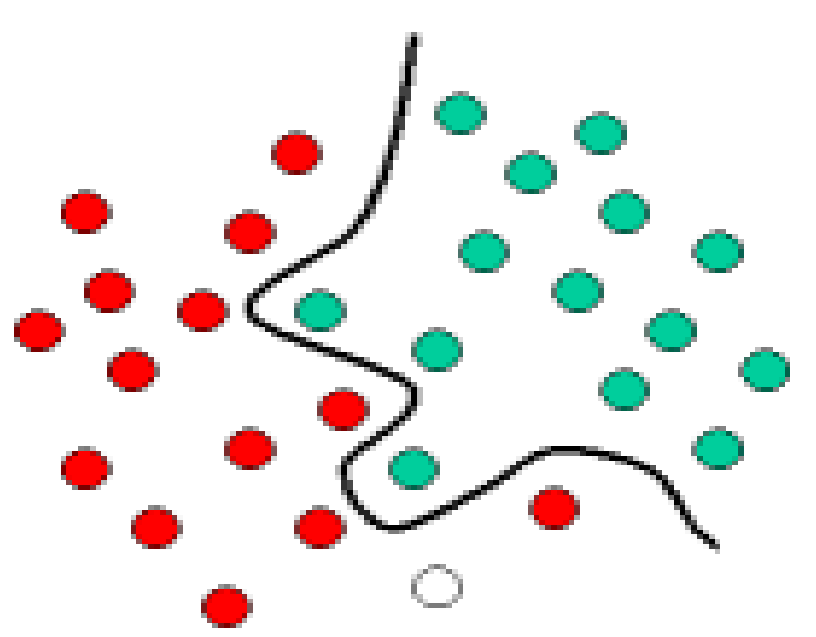

Figure 2: Nonlinear Model

The outside is a normal example of a linear classifier, i.e., a classifier that ruptures a set of objects into their individual groups (GREEN and RED in this state) with a streak. Utmost cataloging shops, hitherto are not that modest, and frequently more compound establishments are obligatory in order to make a perfect parting, i.e., appropriately classify fresh objects (testing) on the basis of the example that are available (training). This national is presented in the diagram Figure 2. Likened to the previous diagram, it is robust that an engaged valedictory of the GREEN and RED objects would essential a curve. Cataloging shops be contingent on draught excruciating lines to distinguish amongst items of numerous lessons relations are recognized as hyperplane classifiers. SVM are mostly right to grip such errands.

The Figure 3 shows the humble imprint late SVM. Now we see the original objects haggard, i.e., rearranged, applying a collection of exact functions, recognized as kernels. The process of regrouping the materials is recognized as registering (change). Letter that in this unique state, the logged objects (right lateral of the drawing) is linearly discrete and, consequently, instead of structure the multiple curve (left diagram), and invention a finest curve that can divided the GREEN and the RED objects [10].

\section{Input space Feature space}

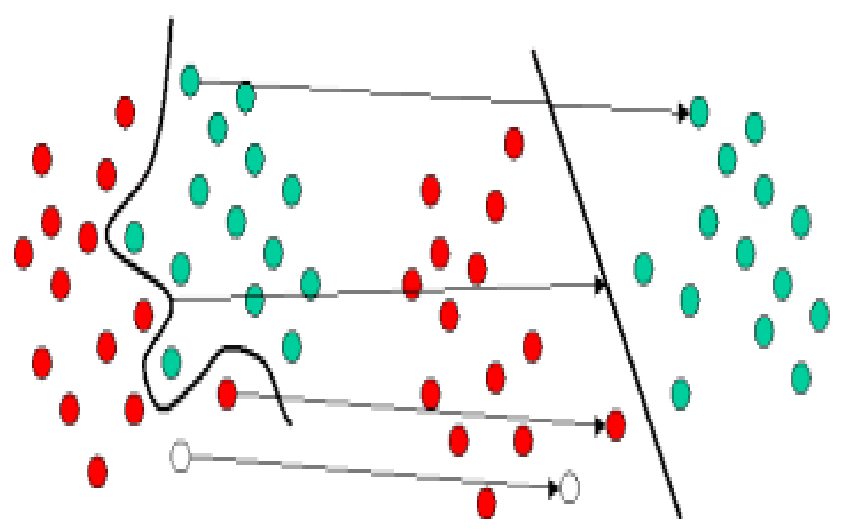

Figure 3: Classification nonlinear model using SVM

\subsection{Power of Signal}

In this chunk fleetingly we will clarify how the forte of a signal is distributed in the frequency domain, relative to the fortes of any signals in the setting, is principal to the plan of entirely linear time-invariant sieve deliberate to suppress or assortment the signal [11]. This decent when signals are deterministic, and it change out to be just as correct in the rank of chance signals. For instance, if a musical waveform is audio signals with cooperative complaint signals, it's essential produce a low pass Linear time-invariant filter for mining the audio and curb the complaint signals [6,12]. Power spectral density function current the power of the energy in the signal as a function of frequency. The component of Power spectral density function is energy (variance) for each frequency(width) and can improvement energy in a sure frequency area by combine Power spectral density in that frequency area [15]. Figure 5 is instance about power spectral for a signal.

\subsection{Proposed Method}

In this section, a new method proposed for High-Energy Physics Problem. The row data first analyzed by using power of signal. The aim of this part is to extracted important features 
from input data and reduce the dimension of data. Then, automatically decrease the computation time which is very important issue when relating with large size of data.

The output of the power of signal wired to the SVM. Then, SVM trained to classify the High-Energy Physics Problem into two classes signal and background. The flowchart of proposed method shown in Figure 4.

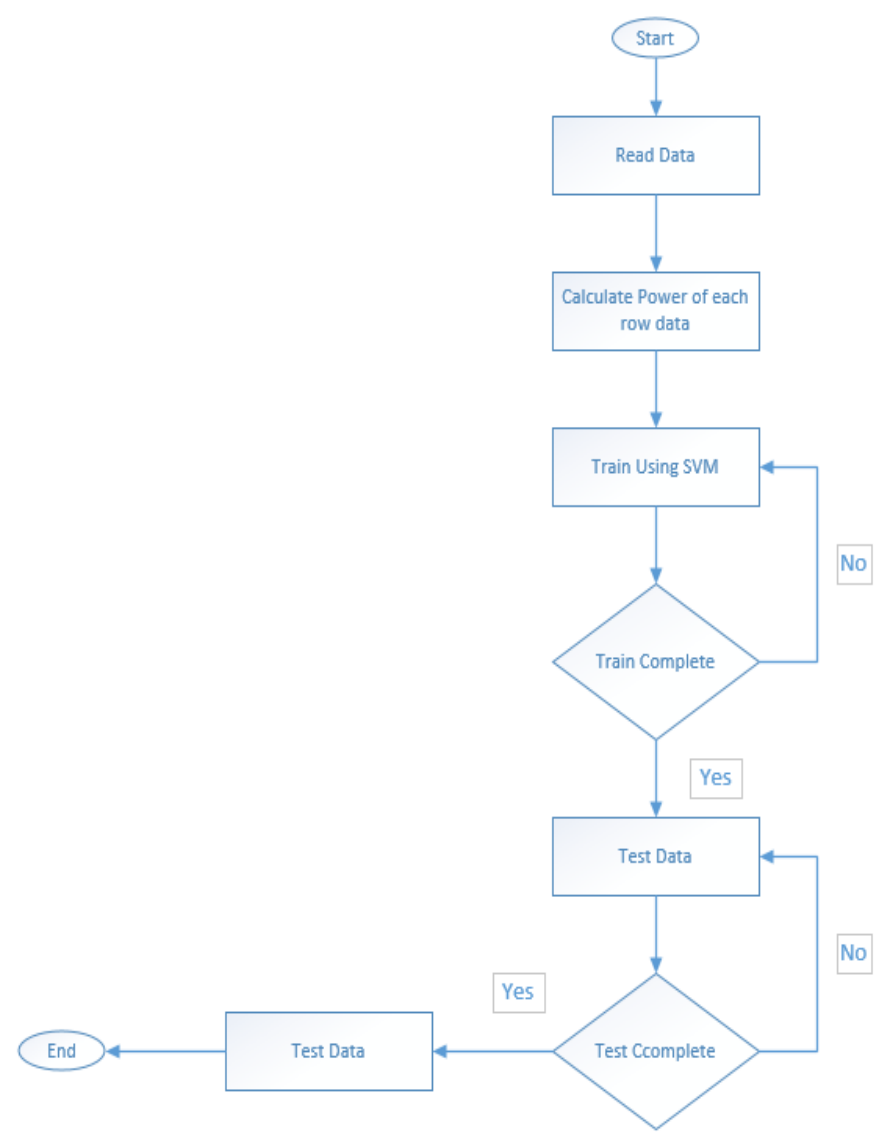

Figure 4: Proposed Method Flowchart

\subsection{High-Energy Physics}

In high energy physics we pursue to comprehend the countryside of space and time, the characteristics of the militaries leading the connections of substance and energy, and the roots of the possessions of the basic atoms. Contemporary theories of particle physics significance to clarify the source of mass, and confidence to unite the explanations of all the militaries, counting seriousness. With the detection that "normal" matter establishes only $4 \%$ of the entire energy in the cosmos, the study of dark matter and dark energy has involved countless attention. Furthermore, dataset published in UCI website used in this paper to validate our method [13]. Then, first column is the lesson label (1 for signal, 0 for background), shadowed by the 27 regularized features ( 22 low-level features then 5 high-level features).

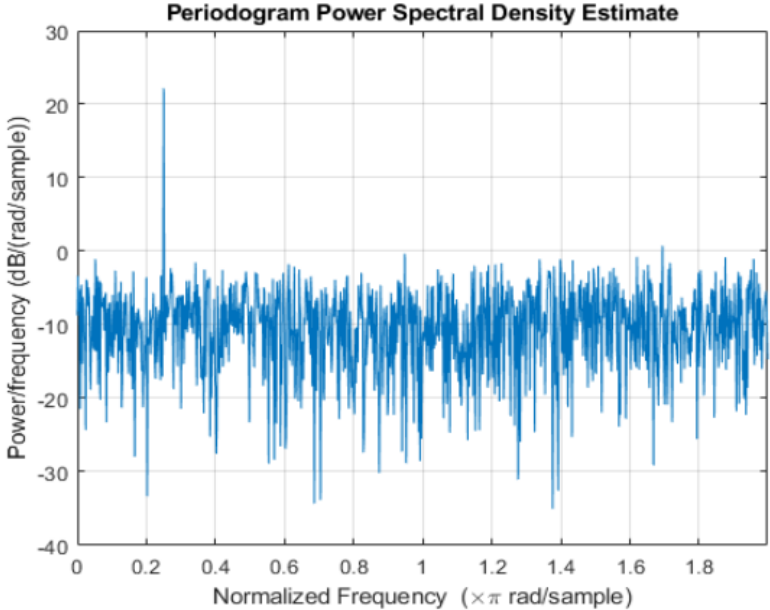

Figure 5: Power Spectral for a Signal.

\section{Results and Discussions}

In this section, experimental results are implemented by using MATLAB 2018, four parameters are calculated to evaluate the performance of proposed method: Accuracy, Sensitivity, Specificity and Precision.

Accuracy $=\frac{(T P+T N)}{(P+N)}$

Sensitivity $=\frac{T P}{(T P+F N)}$

Specificity $=\frac{T N}{(F P+T N)}$

Precision $=\frac{T P}{(T P+F P)}$

To avoid overfitting, the experimental repeated 10 time and the average of these results are calculated and listed in Table 1.

Table 1: Experimental Results

\begin{tabular}{|c|c|}
\hline Parameter & Proposed Method \\
\hline Sensitivity & 1.0000 \\
\hline Sensitivity & 0.8750 \\
\hline Specificity & 0.9884 \\
\hline Accuracy & 0.9893 \\
\hline
\end{tabular}

\section{Conclusions}

This paper proposes a new method for solving High-Energy Physics problem. The proposed method presented remarkable results. Our method presented advantages in time and accuracy by combining the power of signal with SVM which is presented suitable results in various problems. Furthermore, the proposed method can be applied with different classification problems. 


\section{References}

[1] Karim, A. M., Güzel, M. S., Tolun, M. R., Kaya, H., and Çelebi, F. V., "A New Generalized Deep Learning Framework Combining Sparse Auto-encoder and Taguchi Method for Novel Data Classification and Processing," pp. 1-22

[2] Karim, A. M., Çelebi, F. V., and Mohammed, A. S., "Software Development for Blood Disease Expert System," Lect. Notes Softw. Eng., vol. 4, no. 3, pp. 179-183, 2016.

[3] G. Xu and W. Fang, "Shape retrieval using deep Auto-encoder learning representation," 2016 13th Int. Comput. Conf. Wavelet Act. Media Technol. Inf. Process. ICCWAMTIP 2017, pp. 227-230, 2017.

[4] V. Sze, Y.-H. Chen, T.-J. Yang, and J. Emer, "Efficient Processing of Deep Neural Networks: A Tutorial and Survey," vol. 105, no. 12, pp. 2295-2329, 2017.

[5] R. Memisevic, "Deep Learning: Architectures , algorithms , applications," 2015.

[6] A. Luckow, M. Cook, N. Ashcraft, E. Weill, E. Djerekarov, and B. Vorster, "Deep Learning in the Automotive Industry: Applications and Tools," Big Data Int. Conf. Big Data, pp. 3759-3768, 2016.

[7] S. Ibrahim, R. Djemal, and A. Alsuwailem, "Electroencephalography (EEG) signal processing for epilepsy and autism spectrum disorder diagnosis," Biocybern. Biomed. Eng., vol. 38, no. 1, pp. 16-26, 2018.

[8] E. Yavuz, M. C. Kasapbaşı, C. Eyüpoğlu, and R. Yazıcı, "An epileptic seizure detection system based on cepstral analysis and generalized regression neural network," Biocybern. Biomed. Eng., vol. 38, no. 2, pp. 201-216, 2018.

[9] R. Djemili, H. Bourouba, and M. C. Amara Korba, "Application of empirical mode decomposition and artificial neural network for the classification of normal and epileptic EEG signals," Biocybern. Biomed. Eng., vol. 36, no. 1, pp. 285-291, 2016.

[10] J. Martinez-del-Rincon et al., "Non-linear classifiers applied to EEG analysis for epilepsy seizure detection," Expert Syst. Appl., vol. 86, pp. 99-112, 2017.

[11] G. Cottone, M. Di Paola, a new representation of power spectral density and correlation function by means of fractional spectral moments, Prob. Eng. Mech. 25 (2010) 348-353.

[12] Welch, Peter. "The use of fast Fourier transform for the estimation of power spectra: a method based on time averaging over short, modified period grams." IEEE Transactions on audio and electroacoustic 15.2 (1967):70-73.

[13] J. Yang, H. Rivardb and R. Zmeureanua, "On-line building energy prediction using adaptive artificial neural networks", Energy and Buildings, Vol. 92, 2015, pp. 10-18.

[14] Pierre Baldi, Kyle Cranmer, Taylor Faucett, Peter Sadowski, and Daniel Whiteson. 'Parameterized Machine Learning for High-Energy Physics.

[15] Karim, A. M., Güzel, M. S., Tolun, M. R., Kaya, H., \& Çelebi, F. V. (2019). A new framework using deep auto-encoder and energy spectral density for medical waveform data classification and processing. Biocybernetics and Biomedical Engineering, 39(1), 148-159. doi:10.1016/j.bbe.2018.11.004.

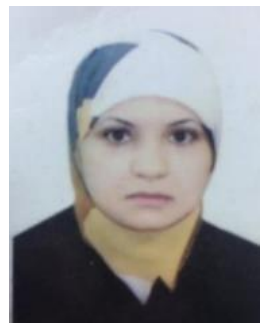

Hind Khudheyer Abbas is a lecturer at University of Karbala, Iraq, in where she was born in Karbala. In 2005, she received her B.Sc. degree in physics from Baghdad University in Baghdad, Iraq. She obtained M.Sc. degree in physics science from Ankara University in Ankara, Turkey, in 2012.

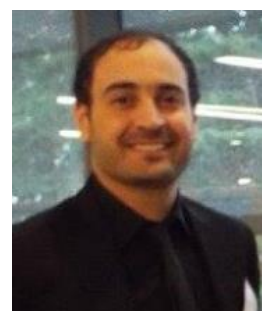

Hisham Jashami obtained his Master of Science in Civil Engineering from Gaziantep University, Turkey in 2012. $\mathrm{He}$ is from Iraq, where he completed his undergraduate in the same area from Al-Mustansiriya University in 2006.

Cite this article as: Hind Khudheyer, Hisham Jashami, Support vector machine based on power of signal for solving high-energy physics problem, International journal of research in engineering and innovation (IJREI), vol 3, issue 1 (2019), 57-60. https://doi.org/10.36037/IJREI.2019.3109 